\title{
Internal wave drag in stratified flow over mountains on a beta plane
}

Article

Accepted Version

Teixeira, M. A. C. and Grisogono, B. (2008) Internal wave drag in stratified flow over mountains on a beta plane. Quarterly Journal of the Royal Meteorological Society, 134 (630). pp. 11 19. ISSN 1477-870X doi: https://doi.org/10.1002/qj.188 Available at https://centaur.reading.ac.uk/29247/

It is advisable to refer to the publisher's version if you intend to cite from the work. See Guidance on citing.

Published version at: http://dx.doi.org/10.1002/qj.188

To link to this article DOI: http://dx.doi.org/10.1002/qj.188

Publisher: Royal Meteorological Society

All outputs in CentAUR are protected by Intellectual Property Rights law, including copyright law. Copyright and IPR is retained by the creators or other copyright holders. Terms and conditions for use of this material are defined in the End User Agreement.

\section{www.reading.ac.uk/centaur}

\section{CentAUR}

Central Archive at the University of Reading

Reading's research outputs online 


\title{
Internal wave drag in stratified flow over mountains on a beta plane
}

\author{
M. A. C. Teixeira ${ }^{\mathrm{a} *}$ and Branko Grisogono ${ }^{\mathrm{b}}$ \\ ${ }^{a}$ University of Lisbon, CGUL, IDL, Lisbon, Portugal \\ ${ }^{\mathrm{b}}$ Department of Geophysics, Faculty of Science, University of Zagreb, Zagreb, Croatia
}

\begin{abstract}
The impact of the variation of the Coriolis parameter $f$ on the drag exerted by internal Rossby-gravity waves on elliptical mountains is evaluated using linear theory, assuming constant wind and static stability, and a beta-plane approximation. The calculations of Smith (1979a) and Miranda and James (1992) of inertia-gravity wave drag are thus extended, in an attempt to establish a connection with existing calculations of planetary wave drag (which have been developed until now primarily for fluids topped by a rigid lid). It is found that the internal wave drag for zonal westerly flow strongly increases relative to that given by the calculation where $f$ is assumed a constant, particularly at high latitudes and for mountains aligned meridionally. For sufficiently wide mountains, the drag increases with the mountain width, taking values much larger than those valid in the non-rotating limit. This occurs because the drag receives contributions from a low wavenumber range, controlled by the beta-effect, which accounts for the drag amplification found here. This drag amplification is shown to be considerable for idealised analogues of real mountain ranges, such as the Himalayas and the Rocky mountains, and comparable to the barotropic Rossby wave drag addressed in previous studies. Copyright (c) 0000 Royal Meteorological Society
\end{abstract}

KEY WORDS Linear theory; Momentum flux; Rossby-gravity waves; Steady state; Vertical propagation

Received 5 March 2007

\section{Introduction}

While the drag force exerted on mountains by internal gravity and inertia-gravity waves has been reasonably well studied (Smith, 1979a; Miranda and James, 1992; Grisogono et al., 1993; Ólafsson and Bougeault, 1997), to the authors' knowledge there are no calculations of the drag in the case of orographic internal Rossby-gravity waves, where the variation of the Coriolis parameter with latitude is important, in a continuously stratified atmosphere. This may be due to the fact that standard internal wave theory uses the Boussinesq approximation, a Cartesian coordinate system and a continuous spectrum of wavenumbers. As pointed out by Smith (1979b), these approximations are no longer accurate for Rossby-gravity waves, since the vertical wavelength of these waves is large, being affected by the vertical density variation in the atmosphere, and horizontally these waves are of planetary scale, being affected by the spherical geometry of the Earth, and possessing a discrete wavenumber spectrum. Additionally, the orographic drag associated with these waves occurs at large scales resolved by even the models with the coarsest horizontal resolutions, such as GCMs, so in general it does not need to be parameterised.

Despite this, most of the studies addressing Rossbygravity waves, and the associated drag (e.g. Janowitz, 1977; McCartney, 1975; Thompson and Flierl, 1993) use a beta-plane approximation, because this is the simplest

\footnotetext{
*Correspondence to: Centro de Geofísica da Universidade de Lisboa, Edifício C8, Campo Grande, 1749-016 Lisbon, Portugal. E-mail: mateixeira@fc.ul.pt
}

way to retain the essential features of Rossby waves, while neglecting other geometrical complications. As a rule, these studies also assume that the fluid under consideration is bounded above by a rigid lid or a sharp density interface. These studies are often concerned with oceanic flows (e.g. Janowitz, 1975), although this approximation may also be viewed as a rough representation of the finite extent of the troposphere (Janowitz, 1977). Consequently, internal waves are either entirely absent (when constantdensity fluids are considered, e.g. Ingersoll, 1969; Thompson and Flierl, 1993) or discarded as insignificant compared to the barotropic mode (Janowitz, 1977), a result that relies crucially on the existence of the rigid lid. The adequacy of this latter choice will be re-examined in the present study.

Smith (1979c) qualitatively mentioned the relevance of the beta-effect on the drag produced by internal Rossby-gravity waves in a study where he analysed the effects of the rigid lid approximation and the 2D approximation. The impacts of these approximations on the surface drag were not addressed, but it is clear that unlike a continuously decreasing density (which in fact can be incorporated into Boussinesq models through a simple change of variables) the assumption of a rigid lid in a stratified atmosphere will modify the internal wave drag considerably. In this situation, wave reflections at the top of the domain will surely originate resonances, in much the same way as described for pure gravity waves in Teixeira et al. (2005) and Teixeira and Miranda (2005).

The assumptions of Boussinesq approximation, semi-infinite continuously stratified atmosphere and a 
continuous wave spectrum may not be strictly accurate in a geophysical context. However, they constitute leadingorder approximations to the problem of internal Rossbygravity waves, and are not much more unrealistic than the assumption of a rigid lid, or of topographic barriers with sharp, or even vertical, edges, such as used by Ingersoll (1969), McCartney (1975) or Thompson and Flierl (1993). On the other hand, these assumptions have the great advantage of facilitating comparison with previous models of gravity and inertia-gravity waves, which adopted them, and for which any connection with existing Rossby-gravity wave drag studies is hard to establish. Thus we believe the assumptions just listed and adopted here are acceptable for tackling our goal - an inclusion of Rossby wave effects in the existing mesoscale drag context.

In the present paper, internal wave drag exerted on an elliptical mountain on a beta-plane is calculated using linear theory assuming, for simplicity, that the atmosphere has constant zonal incoming wind and buoyancy (BruntVäisälä) frequency. This work can be regarded as an extension of the study of Smith (1979a) or the linearised calculations of Miranda and James (1992) to a situation where the Coriolis parameter varies with latitude. Investigating the Rossby-gravity wave drag (RGWD) is relevant from a fundamental point of view, but may also have applications in, e.g., better estimating jet-stream weakening or intensification in future climate scenarios, or even modelling the atmospheric circulation on other planets.

This paper is organised as follows: in section 2 the model, including the governing wave equation and the radiation boundary condition, is introduced. Section 3 presents the main results, namely the dependence of the drag on the dimensionless parameters controlling this problem, and drag calculations for more realistic conditions, using simple approximations to real orography. Finally, section 4 contains the main conclusions of this study.

\section{The vertical structure equation}

Flow over a mountain with an elliptical horizontal crosssection is considered (cf. Phillips, 1984; Ólafsson and Bougeault, 1997; Teixeira and Miranda, 2006). For simplicity, the main axes of the ellipse are assumed to be aligned in the zonal $(x)$ and meridional $(y)$ directions. This kind of orography is able to approximate, to a certain extent, real, anisotropic mountain ranges. The incoming flow is assumed to be constant and in the zonal direction. This flow automatically satisfies the conservation of absolute (or potential) vorticity - if the incoming flow was constant and simultaneously had a meridional component, it would violate this constraint. The flow is also assumed to be steady and in geostrophic and hydrostatic equilibrium, having a westerly orientation, since it is known that Rossby waves only exist for westerly flow.

The basic equations of Thuburn (2006) (his set (1)(6)) are adopted here, but the Boussinesq approximation is further imposed on them. These are the inviscid and adiabatic equations of motion for a beta-plane expressed in spectral space in the horizontal directions. It would at first sight seem questionable to expand the flow variables as Fourier integrals along $x$ and $y$ as is done traditionally in gravity and inertia-gravity wave theories, because of the dependence of $f$ (the Coriolis parameter) on $y$. However, Thuburn (2006) (see also Thuburn and Woolings, 2005) developed an approach for this purpose, which respects the conservation of energy and is thus self-consistent.

The linearised equations of motion take the form:

$$
\begin{aligned}
i U k \hat{u}-f_{0} \hat{v}-i \frac{k \beta}{k^{2}+l^{2}} \hat{u} & =-i \frac{k}{\rho_{0}} \hat{p}, \\
i U k \hat{v}+f_{0} \hat{u}-i \frac{k \beta}{k^{2}+l^{2}} \hat{v} & =-i \frac{l}{\rho_{0}} \hat{p}, \\
-\frac{\hat{p}^{\prime}}{\rho_{0}}+\hat{b} & =0 \\
i U k \hat{b}+N^{2} \hat{w} & =0, \\
i k \hat{u}+i l \hat{v}+\hat{w}^{\prime} & =0,
\end{aligned}
$$

where $U$ and $N$ are the wind velocity and Brunt-Väisälä frequency of the incoming flow, $\rho_{0}$ is a constant reference density, and $(\hat{u}, \hat{v}, \hat{w}), \hat{p}$ and $\hat{b}$ are, respectively, the Fourier transforms of the velocity, pressure and buoyancy perturbations. $\hat{b}=g \hat{\theta} / \theta_{0}$, where $g$ is the acceleration of gravity, $\hat{\theta}$ is the Fourier transform of the potential temperature perturbation and $\theta_{0}$ is a reference potential temperature (assumed to be constant). In (1) and (2), the Coriolis parameter is expressed as $f=f_{0}+\beta y$ (consistent with the beta-plane approximation), where $\beta$ is its meridional derivative. $(k, l)$ is the horizontal wavenumber of the waves and the primes denote differentiation with respect to height, $z$. The third terms is (1) and (2) correspond to the beta-effect, in accordance with the arguments presented by Thuburn and Woolings (2005).

In (3), the hydrostatic approximation was adopted also for the flow perturbations, which is acceptable at the large horizontal scales addressed here. These equations may be combined in order to eliminate all dependent variables except the Fourier transform of the vertical velocity perturbation, $\hat{w}$, yielding an equation akin to the Taylor-Goldstein equation (cf. Teixeira and Miranda, 2006), but where both the effects of $f_{0}$ and $\beta$ are retained:

$$
\hat{w}^{\prime \prime}-\left\{\frac{N^{2}\left(k^{2}+l^{2}\right)\left[1-\frac{\beta}{U\left(k^{2}+l^{2}\right)}\right]}{k^{2} U^{2}\left[1-\frac{\beta}{U\left(k^{2}+l^{2}\right)}\right]^{2}-f_{0}^{2}}\right\} \hat{w}=0 .
$$

In the case of a neutrally stratified atmosphere, such as considered in the study of barotropic Rossby waves by Janowitz (1977), (6) gives the trivial result that $\hat{w}$ varies linearly with height. For $N^{2}>0$, since the coefficient multiplying $\hat{w}$ in (6) is constant, this equation will have solutions of the form

$$
\hat{w}(z)=\hat{w}(z=0) \mathrm{e}^{i m z}
$$


where $m$ is a vertical wavenumber. By direct substitution of (7) in (6), one obtains:

$$
m^{2}=\frac{N^{2}\left(k^{2}+l^{2}\right)\left[1-\frac{\beta}{U\left(k^{2}+l^{2}\right)}\right]}{k^{2} U^{2}\left[1-\frac{\beta}{U\left(k^{2}+l^{2}\right)}\right]^{2}-f_{0}^{2}} .
$$

It may be checked that this definition is consistent with Eq. (58) of Thuburn and Woolings (2005), giving the dispersion relationship of planetary internal waves, if $\omega$ (the angular frequency) is replaced by $-U k$ and the nonBoussinesq terms are neglected. Note that (8) reduces to the usual definition of $m$ for inertia-gravity waves when $\beta=0$, and to the $m$ appropriate for pure gravity waves when both $\beta=0$ and $f_{0}=0$

Equation (6) is subject to two boundary conditions. The lower boundary condition (free-slip condition) requires that the flow be tangential to the topography at the surface, i.e.:

$$
\hat{w}(z=0)=i U k \hat{h},
$$

where $\hat{h}$ is the Fourier transform of the surface elevation. The upper boundary condition requires that the wave energy radiates upwards (since the waves are generated at the surface), and specifies the sign of $m$, which is left undetermined by (8).

For the wave energy to propagate upward, the vertical component of the group velocity of the waves must be positive. The vertical group velocity is defined as $c_{g}=$ $\partial \omega / \partial m$, where $\omega$ is the angular frequency of the waves in a non-stationary version of (6). Although for $\omega=0$, the phase velocity of the waves is zero (because they are by definition stationary), the group velocity is not, and it can be shown that its vertical component consistent with (6) takes the form

$$
\begin{aligned}
& c_{g z}=\frac{2 m U k}{N^{2}\left(k^{2}+l^{2}\right)} \\
& \times \frac{\left\{(U k)^{2}\left[1-\frac{\beta}{U\left(k^{2}+l^{2}\right)}\right]^{2}-f_{0}^{2}\right\}^{2}}{\left[(U k)^{2}+\frac{N^{2}\left(k^{2}+l^{2}\right)}{m^{2}}\right]\left[1-\frac{\beta}{U\left(k^{2}+l^{2}\right)}\right]^{2}+f_{0}^{2}} .
\end{aligned}
$$

It is clear that when $m^{2}>0$ (i.e. the waves are vertically propagating) the denominator of (10) is always positive. Then, the condition that must be satisfied for $c_{g z}>0$ is simply $m U k>0$ in the numerator of (10). So, the radiation boundary condition has the same form as for non-rotating flow (Holton, 2004, Eq. (7.45b)), or rotating flow on an $f$-plane (Wurtele et al., 1996, Eq. (3.2b)), namely:

$$
m=\sqrt{m^{2}} \operatorname{sign}(U k) .
$$

This result will be used in the drag calculations that follow.

\subsection{Wave drag}

The drag exerted on the mountain is defined in Fourier space as (Teixeira and Miranda, 2006)

$$
\left(D_{x}, D_{y}\right)=4 \pi^{2} i \int_{-\infty}^{+\infty} \int_{-\infty}^{+\infty}(k, l) \hat{p}^{*}(z=0) \hat{h} d k d l
$$

where $\hat{p}$ is the Fourier transform of the pressure perturbation and the asterisk denotes complex conjugate.

The pressure perturbation at the surface may be obtained in terms of $\hat{w}$ by combining the equations of motion (1)-(5) and using also (7). If the lower boundary condition (9) is additionally used, this yields:

$$
\hat{p}(z=0)=i \rho_{0} \frac{\left\{(U k)^{2}\left[1-\frac{\beta}{U\left(k^{2}+l^{2}\right)}\right]^{2}-f_{0}^{2}\right\} m \hat{h}}{\left(k^{2}+l^{2}\right)\left[1-\frac{\beta}{U\left(k^{2}+l^{2}\right)}\right]} .
$$

On inserting (13) into (12), and using also (8) and (11) to substitute $m$, the RGWD becomes

$$
\begin{aligned}
& \left(D_{x}, D_{y}\right)=4 \pi^{2} \rho_{0} N U \int_{-\infty}^{+\infty} \int_{\infty}^{+\infty} \frac{(k, l)|\hat{h}|^{2}}{\left(k^{2}+l^{2}\right)^{\frac{1}{2}}} \\
& \times \operatorname{sign}(k) \operatorname{Re}\left\{\frac{k^{2}\left[1-\frac{\beta}{U\left(k^{2}+l^{2}\right)}\right]^{2}-\frac{f_{0}^{2}}{U^{2}}}{1-\frac{\beta}{U\left(k^{2}+l^{2}\right)}}\right\}^{\frac{1}{2}} d k d l,
\end{aligned}
$$

where 'Re' denotes 'real part'.

Since this is the type of orography used in most of the studies that the present calculations aim to extend (e.g. Phillips, 1984; Ólafsson and Bougeault, 1997) the mountain is assumed to have an elliptical horizontal crosssection and a bell-shaped profile:

$$
h=\frac{h_{0}}{\left(1+\frac{x^{2}}{a^{2}}+\frac{y^{2}}{b^{2}}\right)^{\frac{3}{2}}} \Rightarrow \hat{h}=\frac{h_{0} a b}{2 \pi} \mathrm{e}^{-\left(a^{2} k^{2}+b^{2} l^{2}\right)^{\frac{1}{2}}} \text {, }
$$

where $h_{0}$ is the maximum height, $a$ is the zonal halfwidth and $b$ is the meridional half-width. It may be noted that, for this kind of mountain or any other orography that is symmetric with respect to $y$, the corresponding Fourier transform is even in $l$. So the integral of $D_{y}$ cancels in (14) by symmetry, because the integrand is odd in $l$. Consequently, we will only focus on the zonal drag component $D_{x}$, which hereafter will be called just $D$.

Given (15), it is convenient to perform a change of variables in the integrals of (14), adopting polar elliptical coordinates for the horizontal wavenumbers, as follows (cf. Teixeira and Miranda, 2006):

$$
k=\frac{\kappa}{a} \cos \theta, \quad l=\frac{\kappa}{b} \sin \theta
$$

Then, using (15), (14) may be expressed as

$$
\begin{aligned}
& D=\rho_{0} N U b h_{0}^{2} \int_{0}^{2 \pi} \int_{0}^{+\infty} \frac{\kappa \mathrm{e}^{-2 \kappa}|\cos \theta|}{\left(\cos ^{2} \theta+\gamma^{2} \sin ^{2} \theta\right)^{\frac{1}{2}}} \\
& \operatorname{Re}\left\{\frac{\kappa^{2} \cos ^{2} \theta\left[1-\frac{N_{\beta}}{\kappa^{2}\left(\cos ^{2} \theta+\gamma^{2} \sin ^{2} \theta\right)}\right]^{2}-R o^{-2}}{1-\frac{N_{\beta}}{\kappa^{2}\left(\cos ^{2} \theta+\gamma^{2} \sin ^{2} \theta\right)}}\right\}^{\frac{1}{2}} \\
& d \kappa d \theta,
\end{aligned}
$$


where $\gamma=a / b$ is the horizontal aspect ratio of the mountain, $R o=U /\left(f_{0} a\right)$ is a Rossby number and $N_{\beta}=\beta a^{2} / U$ is a dimensionless number quantifying the importance of the beta-effect. A dimensionless number similar to $N_{\beta}$ was called $\hat{\beta}$ by Bannon (1980) and $b$ by McCartney (1975), to give just a few examples.

In order to facilitate its interpretation, the drag is normalised by its value in the absence of rotation, which results from (17) when $R o^{-1}=N_{\beta}=0$ :

$$
D_{0}=\rho_{0} N U b h_{0}^{2} \int_{0}^{2 \pi} \int_{0}^{+\infty} \frac{\kappa^{2} \mathrm{e}^{-2 \kappa} \cos ^{2} \theta}{\left(\cos ^{2} \theta+\gamma^{2} \sin ^{2} \theta\right)^{\frac{1}{2}}} d \kappa d \theta
$$

Another important reference value is the quasigeostrophic drag, to which (17) reduces when $R o \ll 1$ :

$$
\begin{aligned}
& D_{Q G}=\rho_{0} N f_{0} a b h_{0}^{2} \int_{0}^{2 \pi} \int_{0}^{+\infty} \frac{\kappa \mathrm{e}^{-2 \kappa}|\cos \theta|}{\left(\cos ^{2} \theta+\gamma^{2} \sin ^{2} \theta\right)^{\frac{1}{2}}} \\
& \times \operatorname{Re}\left[\frac{N_{\beta}}{\kappa^{2}\left(\cos ^{2} \theta+\gamma^{2} \sin ^{2} \theta\right)}-1\right]^{-\frac{1}{2}} d \kappa d \theta .
\end{aligned}
$$

While in (18) all wavenumbers contribute to the drag, in (19) only the wavenumbers that satisfy

$$
\kappa<\left[\kappa_{1}=\frac{N_{\beta}^{\frac{1}{2}}}{\left(\cos ^{2} \theta+\gamma^{2} \sin ^{2} \theta\right)^{\frac{1}{2}}}\right]
$$

contribute, and in (17) the pattern is still more complex, with wavenumbers both above a certain threshold and contained in a lower interval contributing, namely

$$
\begin{aligned}
& \kappa>\left\{\kappa_{1}^{2}+\kappa_{2}^{2}+\left[\left(\kappa_{1}^{2}+\kappa_{2}^{2}\right)^{2}-\kappa_{1}^{4}\right]^{\frac{1}{2}}\right\}^{\frac{1}{2}} \\
& \text { or }\left\{\kappa_{1}^{2}+\kappa_{2}^{2}-\left[\left(\kappa_{1}^{2}+\kappa_{2}^{2}\right)^{2}-\kappa_{1}^{4}\right]^{\frac{1}{2}}\right\}^{\frac{1}{2}}<\kappa<\kappa_{1},
\end{aligned}
$$

where

$$
\kappa_{2}=\frac{R o^{-1}}{\sqrt{2}|\cos \theta|}
$$

The upper wavenumber range (which vanishes in the quasi-geostrophic limit) clearly corresponds to the drag produced by gravity waves, as in Smith (1979a), Appendix A of Miranda and James (1992) or Grisogono et al. (1993), while the lower wavenumber range accounts for the drag produced by internal Rossby waves. It is interesting how, in contrast, for a non-stratified flow with a rigid lid, all contributions to the drag come from the singular wavenumber where the expression inside square brackets in (19) is zero - the wavenumber of free barotropic Rossby waves (see Janowitz, 1977).

\section{Results and discussion}

It should first be noted that, when $N_{\beta}=0$, e.g. at the poles of the Earth, (17) reduces to

$$
\begin{aligned}
& D=\rho_{0} N U b h_{0}^{2} \int_{0}^{2 \pi} \int_{0}^{+\infty} \frac{\kappa \mathrm{e}^{-2 \kappa}|\cos \theta|}{\left(\cos ^{2} \theta+\gamma^{2} \sin ^{2} \theta\right)^{\frac{1}{2}}} \\
& \times \operatorname{Re}\left(\kappa^{2} \cos ^{2} \theta-R o^{-2}\right)^{\frac{1}{2}} d \kappa d \theta
\end{aligned}
$$

which, for the case of a circular mountain $(\gamma=1)$ is equivalent to Eq. (A15) or Miranda and James (1992). In this case, the incoming flow should be understood as not zonal (which would not make sense at the pole), but rather having any orientation. This is admissible because the absence of the beta-effect removes the anisotropy present in the original problem formulation. A lower cutoff for the horizontal wavenumbers that contribute to the drag is set by the condition

$$
\kappa>\frac{R o^{-1}}{|\cos \theta|}
$$

coming from the requirement that the expression between brackets at the bottom in (24) be positive. For this reason, as the mountain becomes wider (as $R o^{-1}$ increases) the drag decreases rapidly, i.e. approximately exponentially, as in Appendix A of Miranda and James (1992) (and in Smith, 1979a and Grisogono et al., 1993 for a 2D ridge).

When $N_{\beta} \neq 0$, however, a lower range of wavenumbers contribute to the drag, as was seen above. For wide mountains (i.e. $N_{\beta}$ relatively high), this leads to large values of the drag coming from contributions of the integrand near $\kappa=\kappa_{1}$, as will be seen next. In fact, there must be a lower limit imposed on the wavenumbers that contribute to the drag additionally to that expressed by (22), due to the finite dimensions of the Earth. This has a very small impact on the drag. A more stringent limit, since we are considering zonal incoming flows (except at the pole), would be the length of the Earth's parallels at each latitude considered. It was verified that this also has a very modest impact, for latitudes lower than $60^{\circ}$.

Another, more serious, limitation of the present approach is that it does not take into account the discrete nature of the zonal wavenumbers that can exist on the spherical Earth. This aspect is, again, especially relevant in the lowest wavenumber range for which the integral in (17) gives contributions to the drag, (22). While this limitation can probably introduce large errors in geophysically realistic conditions, we decide here to keep the continuous formulation, because it facilitates the treatment of different orography shapes, and the study of asymptotic drag regimes, avoiding the obscuring discontinuities in drag behaviour brought about by a discrete approach. The continuous approach can be considered suitable for a generic planet with very large radius, but qualitatively the behaviour of the RGWD is similar to that occurring when the wavenumbers are discrete.

A rough idea of the impact of this aspect may be obtained by noting that (22) may be expressed in terms of $k$ (the zonal wavenumber), defining an interval for 


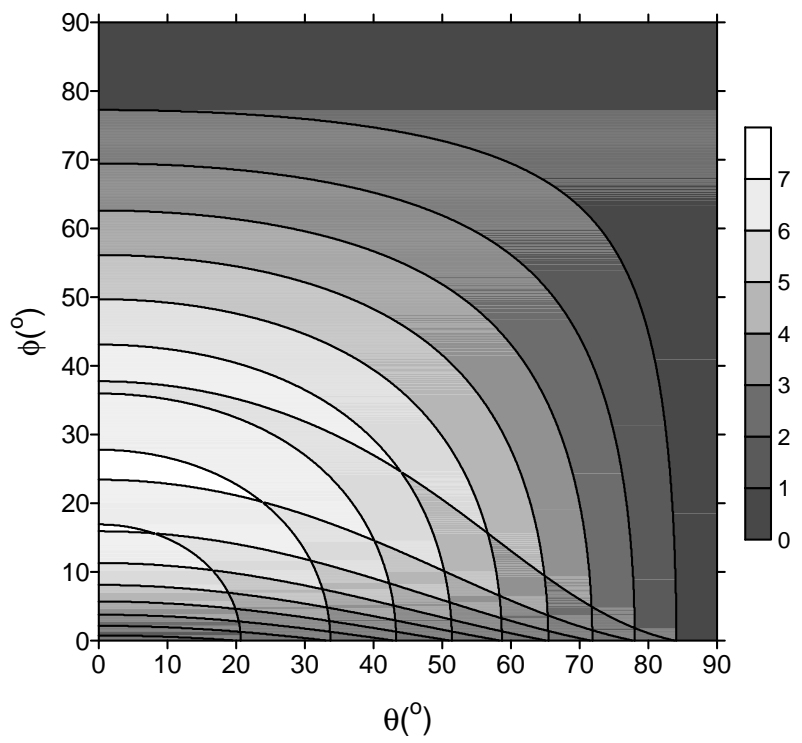

Figure 1. Amount of discrete zonal wavenumbers compatible with the Earth's radius that can exist, at each latitude, and for each wavenumber direction, within the interval defined by (22), for a circular mountain, $\gamma=1$.

which this quantity contributes to the drag. We may note additionally that in the spherical Earth, the zonal wavenumbers that can exist are given by

$$
k=\frac{n}{R_{E} \cos \phi},
$$

where $R_{E}$ is the radius of the Earth, $n$ is an integer number and $\phi$ is the latitude. Figure 1 shows how many of these discrete wavenumbers fit into the interval for $k$ defined by (22), and so that contribute to the RGWD, as a function of $\theta$ (the wavenumber angle) and latitude. A circular mountain, $\gamma=1$, is considered, and $R_{E}=6.37 \times 10^{6} \mathrm{~m}$ and $U=10 \mathrm{~m} \mathrm{~s}^{-1}$ are used as reference values. It is also noted that $f_{0}=2 \Omega \sin \phi$ and $\beta=2 \Omega / R_{E} \cos \phi$, where $\Omega$ is the angular velocity of rotation of the Earth. It is seen that the amount of possible discrete wavenumbers is zero both for $\phi=0^{\circ}$ and $\phi=90^{\circ}$, or when $l$ is very large $\left(\theta \approx 90^{\circ}\right)$, because the amplitude of the interval defined by (22) goes to zero in these limits. For $\theta=0$, the amount of discrete wavenumbers is a maximum and goes from 6 at $\phi=30^{\circ}$ to 3 at $\phi=60^{\circ}$, reaching an absolute maximum of 7 slightly below $\phi=30^{\circ}$. So the results to be presented at higher latitudes should be less reliable because of the assumed continuous spectrum.

\subsection{RGWD as a function of $R o^{-1}$ and $N_{\beta}$}

Figure 2 shows the RGWD (17) normalised by its nonrotating value (18) in $\left(R o^{-1}-N_{\beta}\right)$ parameter space, for $R o^{-1}$ between $10^{-2}$ and $10^{2}$ and $N_{\beta}$ between $10^{-6}$ and $10^{2}$. Only the case of a circular mountain was considered $(\gamma=1)$, because differences in drag behaviour for other values of $\gamma$ were found not to be very striking in this representation. Anisotropic mountains will be considered later. The solid lines are contours of the normalised drag,

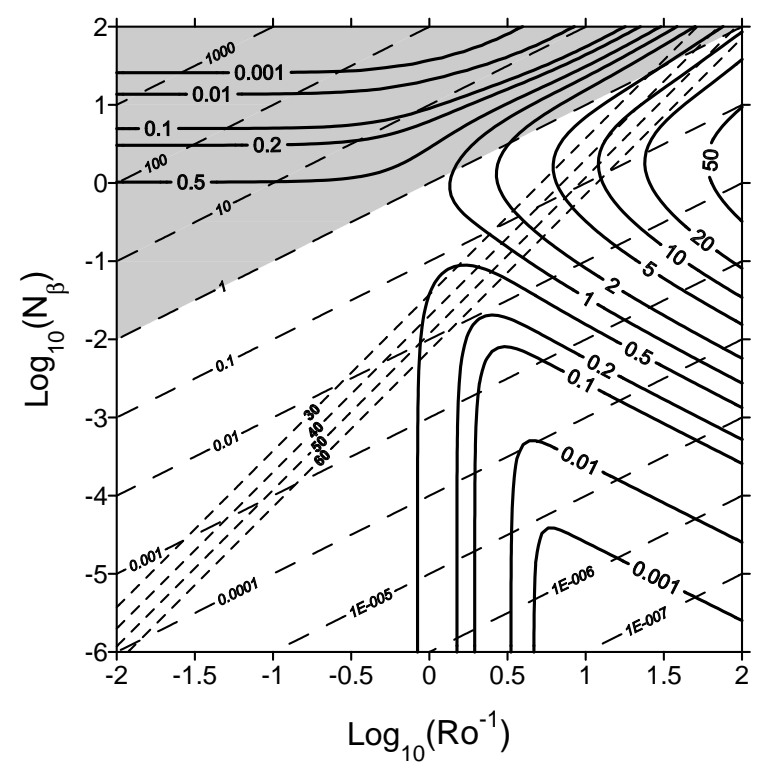

Figure 2. Normalised RGWD as a function of $R o^{-1}$ and $N_{\beta}$ for a circular mountain, $\gamma=1$. Solid lines: labelled contours, short-dashed lines: $U=10 \mathrm{~m} \mathrm{~s}^{-1}$ for different latitudes in degrees (labelled), long-dashed lines: parameter $\varepsilon$ as given by (27). Grey Shading: region where $\varepsilon>1$.

while the long dashed lines represent values of the ratio

$$
\varepsilon=\frac{\beta b}{f_{0}}=\frac{N_{\beta}}{\gamma R o^{-1}},
$$

which must be at most of order one for the beta-plane approximation to be valid. For this reason, in the region with the grey shading (where $\varepsilon>1$ ), the results are deemed to be unreliable. The short-dashed lines illustrate conditions for flows with $U=10 \mathrm{~m} \mathrm{~s}^{-1}$, at latitudes of $30^{\circ}, 40^{\circ}, 50^{\circ}$ and $60^{\circ}$ (these relations are independent of the ridge width $a$, because they have been obtained eliminating this quantity between $R o^{-1}$ and $N_{\beta}$ ). As can be seen, for this range of latitudes and values of $R o^{-1}$ and $N_{\beta}$, the short-dashed lines generally stay outside the shaded region, indicating that the beta-plane approximation is essentially valid. For lower values of $U$, this condition is satisfied even more closely, while for higher $U$, or for lower latitudes, the opposite happens.

It can be seen that the drag is near one for low values of $R o^{-1}$ and $N_{\beta}$, as expected. On the other hand, when $R o^{-1}$ is large, the drag takes high values. These values are largest when $N_{\beta}=O(1)$, being somewhat smaller when either $N_{\beta} \ll 1$ or $N_{\beta} \gg 1$. The existence of high values of the drag due to the beta-effect is a well-known result since early studies on planetary waves, and is illustrated neatly, for example, in Thompson and Flierl (1993) for barotropic Rossby waves in a neutrally stratified and vertically bounded fluid. In the present study, this drag amplification can be explained by making the quasi-gestrophic approximation, which corresponds to taking $R o^{-1} \rightarrow+\infty$ in (17). The RGWD then takes the asymptotic form (19), which when normalised by (18) is proportional to $R o^{-1}$, thus growing indefinitely as this parameter increases. 
When $N_{\beta}$ is small, the drag takes values below 1 for $R o^{-1}=O(10)$. This is a manifestation of the processes that dominate the mesoscale drag in the study of Smith (1979a) or in Appendix A of Miranda and James (1992), however this is not immediately recognisable, because the drag behaviour as $N_{\beta}$ tends to zero, (24), is approached here gradually, particularly for high $R^{-1}$. In fact, it can be shown from (19) that, in the limits $R O^{-1} \rightarrow+\infty$ and $N_{\beta} \rightarrow 0$, the normalised RGWD is asymptotically

$$
\frac{D_{Q G}}{D_{0}} \sim 4 R o^{-1} N_{\beta}
$$

for a circular mountain $(\gamma=1)$. It suffices to note that, when $N_{\beta}$ is small, the exponential in (19) is approximately one and the remaining terms may be integrated directly, yielding the desired result.

In this same limit, the barotropic Rossby wave drag calculated by McCartney (1975) and Janowitz (1977, his Eq. (6)), when normalised by (18) for $\gamma=1$, can be shown to take the approximate form:

$$
\frac{D_{B}}{D_{0}} \approx \frac{U}{N H} R o^{-2} N_{\beta}^{\frac{1}{2}},
$$

where $H$ is the depth of the troposphere in Janowitz's notation. From (28) and (29), the ratio of the internal and barotropic drags (ignoring proportionality constants) may be estimated as follows:

$$
\frac{D_{Q G}}{D_{B}} \approx \frac{N H}{U} R o N_{\beta}^{\frac{1}{2}}=\frac{N H}{f_{0}}\left(\frac{\beta}{U}\right)^{\frac{1}{2}}
$$

(see analogous relation near the top of pg. 805 in Janowitz, 1977). Taking values of these parameters typical of mid latitudes, such as $H=10^{4} \mathrm{~m}, f_{0}=10^{-4} \mathrm{~s}^{-1}, N=$ $10^{-2} \mathrm{~s}^{-1}, U=10 \mathrm{~ms}^{-1}$ and $\beta=10^{-11} \mathrm{~s}^{-1} \mathrm{~m}^{-1}$, this ratio takes the value 1 , indicating that internal wave drag may well be of comparable magnitude to the barotropic wave drag. This most likely also happens when $N_{\beta}=$ $O(1)$ (see discussion in Janowitz, 1977), which partially motivates the relevance of the present calculations. In other parameter ranges, the relative magnitude of internal and barotropic drag depends more strongly on the shape of the orography, so it is not so easy to evaluate.

\subsection{RGWD as a function of mountain width}

It is of geophysical relevance to consider the variation of the RGWD with the mountain width for realistic values of $R o^{-1}$ and $N_{\beta}$. This will be done next on noting the definitions of these two dimensionless parameters, and also that $f=2 \Omega \sin \phi$ and $\beta=2 \Omega / R_{E} \cos \phi$. The situations considered will be those represented as the short-dashed lines in Figure 2, but now for mountains with different aspect ratios.

Figure 3(a) shows the normalised RGWD, given by (17) over (18), as a function of zonal mountain halfwidth, for an incoming wind of magnitude $U=10 \mathrm{~m} \mathrm{~s}^{-1}$ and latitudes between $\phi=30^{\circ}$ and $\phi=60^{\circ}$, for $\gamma=0.25$,
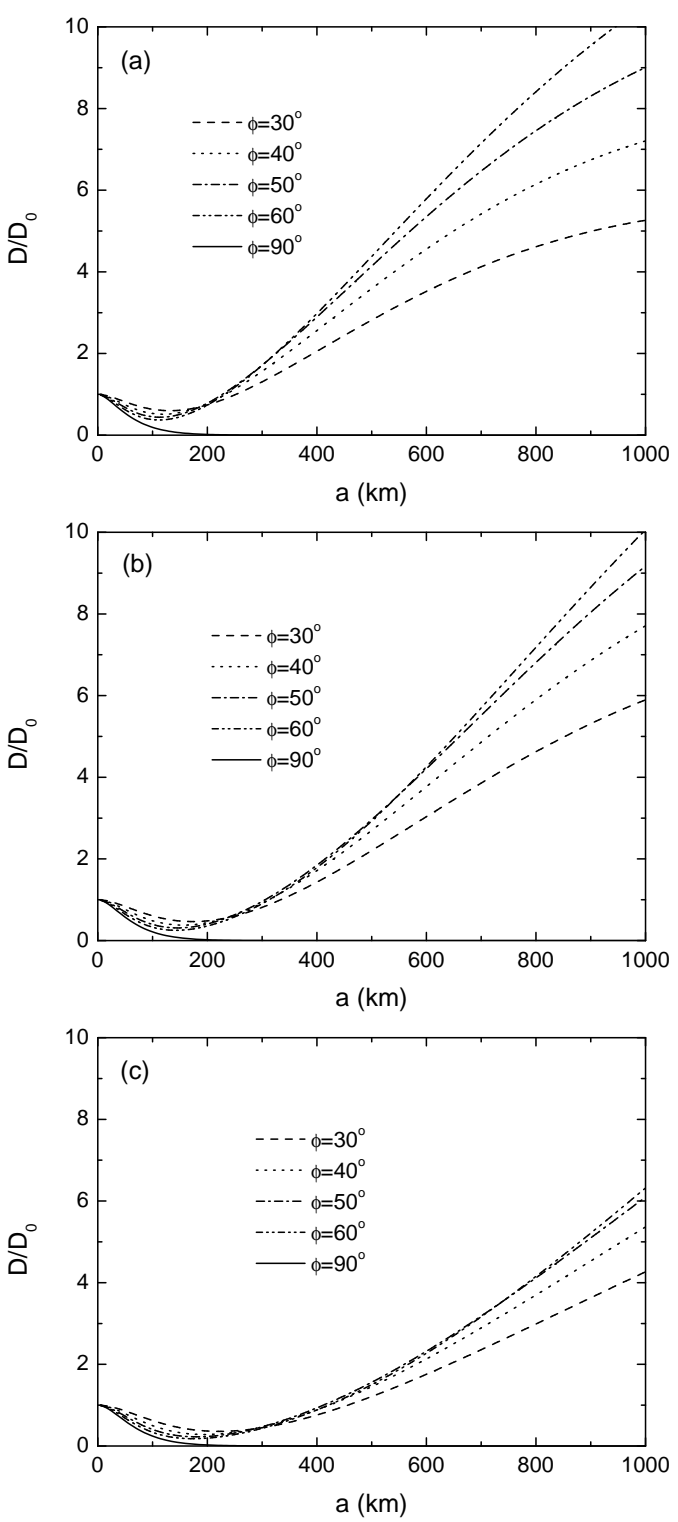

Figure 3. Normalised RGWD as a function of the zonal half-width of the mountain for flow with $U=10 \mathrm{~m} \mathrm{~s}^{-1}$, and various latitudes $\left(\phi=90^{\circ}\right.$ corresponds to (24)). See legend for details. (a) Mountain aligned meridionally, $\gamma=0.25$, (b) circular mountain, $\gamma=1$, (c) mountain aligned zonally, $\gamma=4$.

i.e. a meridional ridge perpendicular to the wind. The RGWD for a latitude $\phi=90^{\circ}$, which is given by (24) and reduces to the linear result of Miranda and James (1992) when $\gamma=1$, is also represented. It can be seen that, as latitude increases between $30^{\circ}$ and $60^{\circ}$, the RGWD tends to be amplified progressively more as the mountain width increases, attaining values more than 10 times larger than that of the drag without rotation for $a=1000 \mathrm{~km}$. A qualitatively similar behaviour was observed for very different conditions (a bounded, neutrally stratified flow over a cylindrical mountain) by Thompson and Flierl (1993). The RGWD attains a minimum between $a=100$ $\mathrm{km}$ and $a=200 \mathrm{~km}$, due to the competing effects of $f_{0}$ and $\beta$. On the other hand, when $a>200 \mathrm{~km}$, the drag is many orders of magnitude larger than given by the 
expression obtained in Appendix A of Miranda and James (1992).

In Figs. 3(b) and 3(c), similar results are shown, but for $\gamma=1$ (a circular mountain) and $\gamma=4$ (a zonally oriented mountain, parallel to the flow), respectively. It can be seen that, as could perhaps be anticipated, the drag increase is more modest for wind along the mountain than across the mountain, for a similar zonal half-width. This happens because, the lower $\gamma$ is, the larger is the meridional extent of the mountain, thereby leading to a greater deflection of the flow and the generation of more intense Rossby-gravity waves.

As latitude increases, a point must be reached where the RGWD starts decreasing toward its low polar values (due to the narrowing of the wavenumber interval (22) that contributes to the drag in (17)). The parameter regime where the drag is stationary with $\phi$ before it begins decreasing seems to occur around $\phi=50^{\circ}$ or $\phi=60^{\circ}$ in Fig. 3(c), but appears to occur only at higher latitudes for lower values of $\gamma$, as shown in Figs. 3(a) and 3(b).

\subsection{Representative orography}

In order to better illustrate the practical relevance of the calculations developed here, two examples of the RGWD produced by approximations to real mountain ranges will be presented: the Himalayas and the Rocky Mountains. The surface elevation for both mountain ranges was taken from the US Navy elevation database. The Himalayas are arbitrarily defined as the region between $62^{\circ} \mathrm{E}$ and $107^{\circ}$ E longitude and between $23^{\circ} \mathrm{N}$ and $40^{\circ} \mathrm{N}$ latitude. The Rocky mountains, on the other hand, are assumed to exist between $116^{\circ} \mathrm{W}$ and $101^{\circ} \mathrm{W}$ longitude and $32^{\circ} \mathrm{N}$ and $47^{\circ}$ $\mathrm{N}$ latitude. The surface elevation distribution contained in these boxes is then centred and adjusted, through a leastsquares fit, to an idealised mountain with Gaussian shape:

$$
h=h_{0} \exp \left(-\frac{x^{2}}{a^{2}}-\frac{y^{2}}{b^{2}}\right) .
$$

The Fourier transform of this surface elevation then replaces (15) in the drag expressions.

This type of orography is used here rather than the bell-shaped mountain employed previously, since the latter was judged to be too spiky to provide a reasonable fit to the mountain ranges under consideration. Note also that (31) still assumes a meridional or zonal orientation of the mountain ranges, which is not too bad for the Himalayas, which are approximately zonal, or for the Rockies, which are approximately meridional. Finally, consistent with the beta-plane approximation, the geometry of the level terrain was approximated as Cartesian, with zonal displacements converted from degrees to distances, assuming a mean latitude $\phi_{0}$.

Table I shows that, for a wind speed $U=10 \mathrm{~m} \mathrm{~s}^{-1}$ or $U=20 \mathrm{~ms}^{-1}$, the impact of the beta-effect is quite important for the RGWD exerted on both mountain ranges, making it take values considerably larger than the non-rotating limit. It is also interesting to note that the quasi-geostrophic approximation is quite accurate, surprisingly being better for the Rocky Mountains than for the Himalayas. This may be due to the following reasons: although $R o^{-1}$ is larger for the Himalayas than for the Rockies, $N_{\beta}$ is also larger by a bigger factor. When (17) reduces to (19) in the quasi-geostrophic approximation, the term that must be neglected involves $N_{\beta}$, therefore the larger this parameter is, the poorer this approximation will be. In this sense, the quasi-geostrophic approximation applied to (17) not only requires that $R o \ll 1$, but also roughly that $N_{\beta} \ll R o^{-1}$ and $N_{\beta} \ll R o^{-2}$. Clearly, these conditions are better satisfied by the Rocky Mountains than by the Himalayas (see Table I).

These results, despite all the cautions entailed by the strong approximations made, suggest that the internal RGWD is a highly relevant force, and perhaps not surprisingly, that it can be treated accurately in a quasigeostrophic framework.

\section{Concluding remarks}

The present study has shown that the drag due to internal Rossby-gravity waves existing in a stably stratified westerly flow is not only much larger than the equivalent inertia-gravity wave drag (Smith, 1979a; Ólafsson and Bougeault, 1997), but also larger than the equivalent pure gravity wave drag (Phillips, 1984). Additionally, it is typically comparable with the barotropic wave drag existing in a neutral but vertically bounded atmosphere (Janowitz, 1977). The drag attains a maximum enhancement for large $R o^{-1}$ and $N_{\beta}$ of order one. It is also larger for meridional mountains than for zonal ones, as would perhaps be expected. The RGWD is found to increase with mountain width and latitude, at least for $\phi$ between 30 and 60 degrees.

This force, along with the barotropic Rossby wave drag, may significantly enhance the zonal torque exerted on the Earth by Rossby-gravity waves, contributing to a deceleration of the jet-streams, or the mean westerly flow in general, in mid-latitudes. Admittedly, internal Rossby-gravity waves are resolved by most meteorological models running at the current resolutions except quasi-geostrophic models or climate models of intermediate complexity. However, their existence, which seems to have been overlooked (except for brief references by Smith, 1979c and Janowitz, 1977), must be taken into account in the global angular momentum budget.

The calculations presented in this paper aim to provide a leading-order treatment of a problem which appears to the authors not to have been addressed before. They are probably of more qualitative than quantitative value. Obviously, many improvements and extensions to this treatment are possible. In particular, a discrete spectrum of zonal wavenumbers could replace the continuous one used here. The Boussinesq approximation could also be abandoned by using a procedure analogous to that described, for example, by Smith (1979b, pg. 95). It would presumably be more difficult (and probably less necessary) to abandon the beta-plane approximation in favour of an 
Table I. Input and output parameters for the Himalayas and the Rocky Mountains: Mean latitude, zonal half-width, meridional halfwidth, aspect ratio, beta-plane small parameter, incoming wind velocity, inverse Rossby number, beta-effect number, drag normalised by non-rotating value and drag normalised by quasi-geostrophic value.

\begin{tabular}{|l|r|r|r|r|r|r|r|r|r|r|}
\hline Range & $\phi_{0}\left({ }^{\circ} \mathrm{N}\right)$ & $a(\mathrm{~km})$ & $b(\mathrm{~km})$ & $\gamma$ & $\varepsilon$ & $U\left(\mathrm{~m} \mathrm{~s}^{-1}\right)$ & $R o^{-1}$ & $N_{\beta}$ & $D / D_{0}$ & $D / D_{Q G}$ \\
\hline Himalayas & 33 & 2597 & 959 & 2.708 & 0.2318 & 10 & 20.57 & 12.91 & 11.75 & 0.964 \\
& & & & & & 20 & 10.29 & 6.46 & 6.77 & 0.962 \\
\hline Rockies & 39 & 997 & 1297 & 0.769 & 0.2514 & 10 & 9.13 & 1.764 & 7.21 & 0.991 \\
& & & & & & 20 & 4.563 & 0.882 & 2.598 & 0.987 \\
\hline
\end{tabular}

explicit treatment of the spherical geometry of the Earth. Nevertheless, all these developments (some of which have already been pursued elsewhere for neutrally stratified stratified fluids topped by a rigid lid or a free surface) remain open to further investigation. We must emphasise, however, that our goal was merely to give a flavour of the inclusion of internal Rossby-gravity waves in the existing mesoscale drag context.

\section{Acknowledgements}

We thank Dr. Pedro Miranda and two anonymous referees for useful comments on this paper. M.A.C.T. was supported by Fundação para a Ciência e Tecnologia (FCT) under grant SFRH/BPD/3533/2000 and project BOSS, contract POCTI/CTE-ATM/58932/2004, co-financed by the European Union under program FEDER. B.G. was supported by the Ministry of Science, Education and Sports of the Republic of Croatia (project BORA, no. 1191193086-1311).

\section{References}

Bannon PR. 1980. Rotating barotropic flow over finite isolated topography. J. Fluid Mech. 101: 281-306.

Grisogono B, Pryor SC, Keislar RE. 1993. Mountain wave drag over double bell-shaped orography. Q. J. R. Meteorol. Soc. 119: 199-206.

Holton JR. 2004. An Introduction to Dynamic Meteorology, 4th Edition, Academic Press.

Ingersoll AP. 1969. Inertial Taylor columns and Jupiter's great red spot. J. Atmos. Sci. 26: 744-752.

Janowitz GS. 1975. The effect of bottom topography on a stratified flow in the Beta plane. J. Geophys. Res. 80: 4163-4168.

Janowitz GS. 1977. The barotropic Rossby wave drag due to an isolated topography. J. Atmos. Sci. 34: 802-805.

McCartney MS. 1975. Inertial Taylor columns on a beta plane. J. Fluid Mech. 68: 71-95.

Miranda PMA, James IN. 1992. Non-linear three dimensional effects on the wave drag: splitting flow and breaking waves. Q. J. R. Meteorol. Soc. 118: 1057-1081.

Ólafsson H, Bougeault P. 1997. The effect of rotation and surface friction on orographic drag. J. Atmos. Sci. 54: 193-210.

Phillips DS. 1984. Analytical surface pressure and drag for linear hydrostatic flow over three-dimensional elliptical mountains. J. Atmos. Sci. 41: $1073-1084$.

Smith RB. 1979a. The influence of the Earth's rotation on mountain wave drag. J. Atmos. Sci. 36: 177-180.

Smith RB. 1979b. The influence of mountains on the atmosphere. Adv. Geophys. 21: 87-230.

Smith RB. 1979c. Some aspects of the quasi-geostrophic flow over mountains. J. Atmos. Sci. 36: 2385-2393.

Teixeira MAC, Miranda PMA. 2005. Linear criteria for gravity-wave breaking in resonant stratified flow over a ridge. Q.J.R. Meteorol. Soc. 131: 1815-1820.

Teixeira MAC, Miranda PMA. 2006. A linear model of gravity wave drag for hydrostatic sheared flow over elliptical mountains. $Q$. J. $R$. Meteorol. Soc. 132: 2439-2458.
Teixeira MAC, Miranda PMA, Argain, JL, Valente, MA. 2005. Resonant gravity wave drag enhancement in linear stratified flow over mountains. Q. J. R. Meteorol. Soc. 131: 1795-1814.

Thompson L, Flierl GR. 1993. Barotropic flow over finite isolated topography: steady solutions on the beta-plane and the initial value problem. J. Fluid Mech. 250: 553-586.

Thuburn J. 2006. Vertical discretizations giving optimal representation of normal modes: Sensitivity to the form of the pressure-gradient term. Q. J. R. Meteorol. Soc. 132: 2809-2825.

Thuburn J, Woollings TJ. 2005. Vertical discretizations for compressible Euler equation atmospheric models giving optimal representation of normal modes. J. Comput. Phys. 203: 386-404.

Wurtele MG, Datta A, Sharman RD. 1996. The propagation of a gravityinertia wave in a positively sheared flow. J. Atmos. Sci. 57: 37033715. 